\title{
The Comparative Estimation of MRET Treated and Regular Water Absorption by Human Tissue with the Electro Physical Method
}

\section{Mark Krinker2, Igor Smirnov¹ , Galina Pana ${ }^{3}$}

${ }^{1}$ Global Quantech Inc

${ }^{2}$ Mark Krinker, City College of Technology, CUNY

${ }^{3}$ Galina Pana, Bgmetrix corp

\section{Introduction:}

Drinking water results in gradual penetrating it into human cells and inter-cells fluid.

Depending on the physical quality of the water and its compatibility with a certain organism, dynamics of this process has different rates.

Physical process in water-containing system can be studied using a complex dielectric permittivity.

This is a complex value:<smiles></smiles>

Its real component characterizes an ability of particle to follow external alternating electric field, while the imaginable portion relates to lagging the polarization processes due to structural features of the object. The majority of the polarization processes are those of relaxation type characterized with the relaxation time $\tau_{i}$ which is an individual characteristic of a certain structure. Due to an evitable statistic dispersion of $\tau$, even among same class of the relaxation component, it's possible that not all of them can follow the external field as the external frequency increases, what results in their collision and emanation of the heat called dielectric losses.

For each the type of the relaxation component having the relaxation time $\tau_{I}$ maximum of the dielectric losses takes a place at the frequency

$$
\omega_{i}=\frac{1}{\tau_{i}}
$$

This is a frequency where value of $\varepsilon$ " reaches its maximum. If the studied object is composed of a set of various $\tau$, this will result in origination of the set of the corresponding energy losses bands.

On the other hand, as the frequency of the external field increases, it results in leaving the polarization process by those objects which can't follow the polarization due to their relaxation time $\tau$. As a result, the dielectric permittivity $\varepsilon$ ' decrease as the frequency increases, Fig. 1.

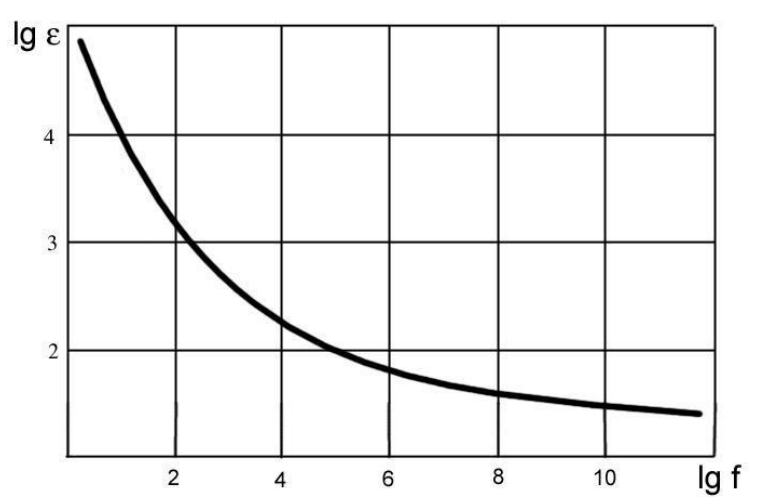

Fig.1. Generalized frequency dependence of the real part of the dielectric permittivity of a leaf

In this brief research, the water absorption was studied as a variation of the complex dielectric permittivity $\varepsilon$ " with Q-meter method and instrument at the fixed frequency of $40 \mathrm{kHz}$.

This method and the instrument enable to measure components of the complex dielectric permittivity, which characterizes polarization processes in alternating field as an indicator of structural patterns of molecular/atomic aggregates.

The Q-meter is a resonance instrument where the quality of the object, the Q-factor (the merit factor) and the frequency allocation of the resonant process of the instrument depends on the connected external capacitance containing the studied object.

This article is published under the terms of the Creative Commons Attribution License 4.0

Author(s) retain the copyright of this article. Publication rights with Alkhaer Publications.

Published at: http://www.ijsciences.com/pub/issue/2017-05/

DOI: 10.18483/ijSci.1304; Online ISSN: 2305-3925; Print ISSN: 2410-4477 
The dissipation factor $\varepsilon^{\prime \prime}=\varepsilon^{\prime} \tan \delta$. For measurements with the Q-meter,

$\varepsilon^{\prime}=\left(C_{0}-C_{x}\right) / C_{0}$, where $C_{x, 0}$ are values of the $\mathrm{Q}$ meter capacitor at the resonance with object $\mathrm{X}$ and without it, respectively. Here: $\tan \delta-\mathrm{a}$ tangent of dielectric loss angle.

The basic formula of the Q-meter measurements:

$$
\tan \delta=\frac{C_{o}\left(Q_{0}-Q_{x}\right)}{\left(C_{0}-C_{x}\right)\left(Q_{0} Q_{x}\right)}
$$

\section{Method and Materials:}

The experiment on measuring water absorption was conducted with TESLA BM-560

Q-meter. Simplified allocation of the setup is shown in Fig.2.

In the experiment, the participant drunk $250 \mathrm{ml}$. of water of $10 \mathrm{C}^{\mathrm{o}}$ temperature, either regular water, or specially MRET activated one. Then the participant put his/her hand on the plate sensor and the reading of the resonant variable capacitance $\mathrm{C}$ of the instrument and its $\mathrm{Q}$ were measured, based on the reading of the voltmeter $\mathrm{V}$. The variable capacitor $\mathrm{C}$ had $0.1 \mathrm{pF}$ for the division. Each reading was taken in one minute. For studying the dynamic of the process, it's not necessary to measure a real dielectric permittivity, but rather the variation of the capacitance $C_{0}-C_{x}$ when the hand was put on the plate-sensor.

Moreover, the deviation of this value was measuring with respect to the initial capacitance $C_{0}-C_{x}$ before drinking the water. So, the very first reading for the capacitance in all the diagrams is zero.

The relative dynamic of the dissipation factor was estimated as a product of the variation of the capacitance and the tangent of dielectric loss angle.

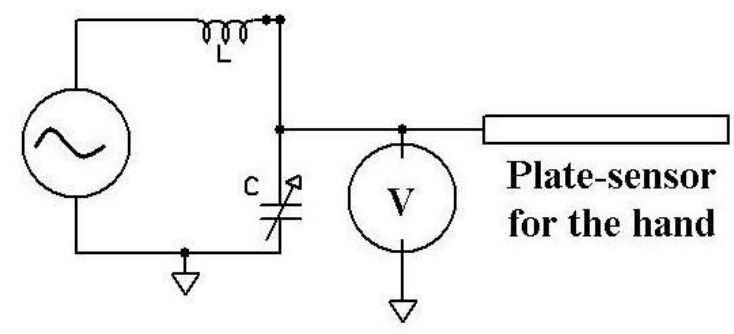

Fig.2. A simplified setup for measuring a dynamics of the water absorption with Q-meter

\section{Results:}

Isabella. Regular Water

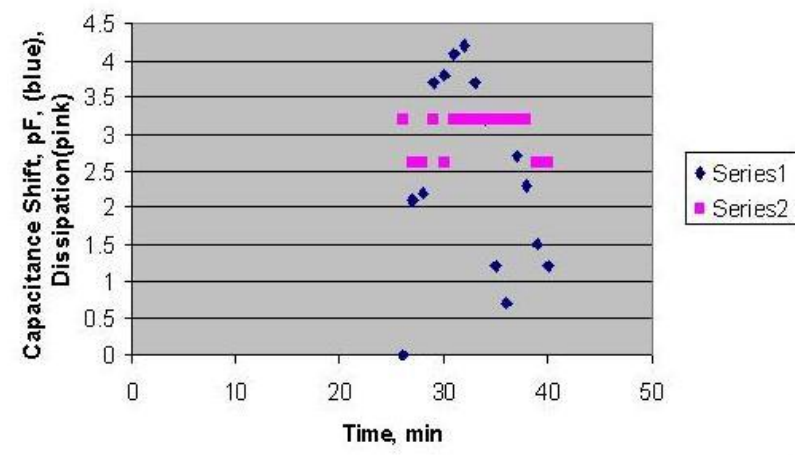

Fig.3. Initial capacitance $=85.3 \mathrm{pF}$

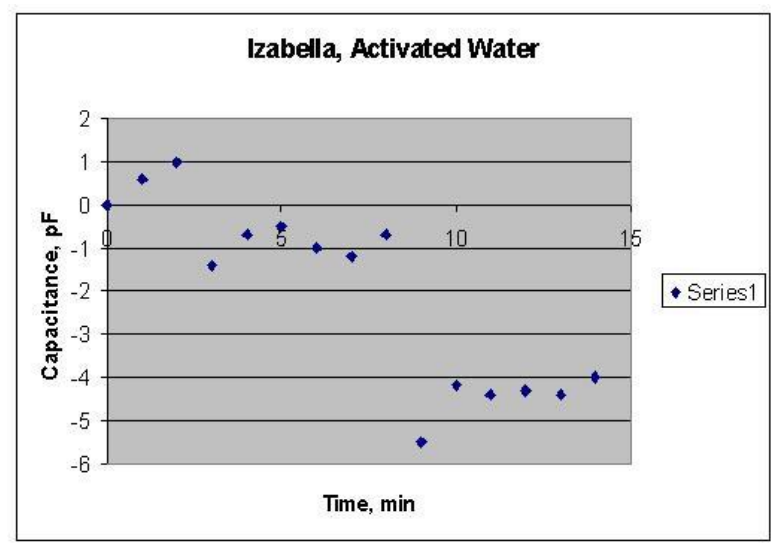

Fig.4. Initial capacitance $=87.4 \mathrm{pF}$.

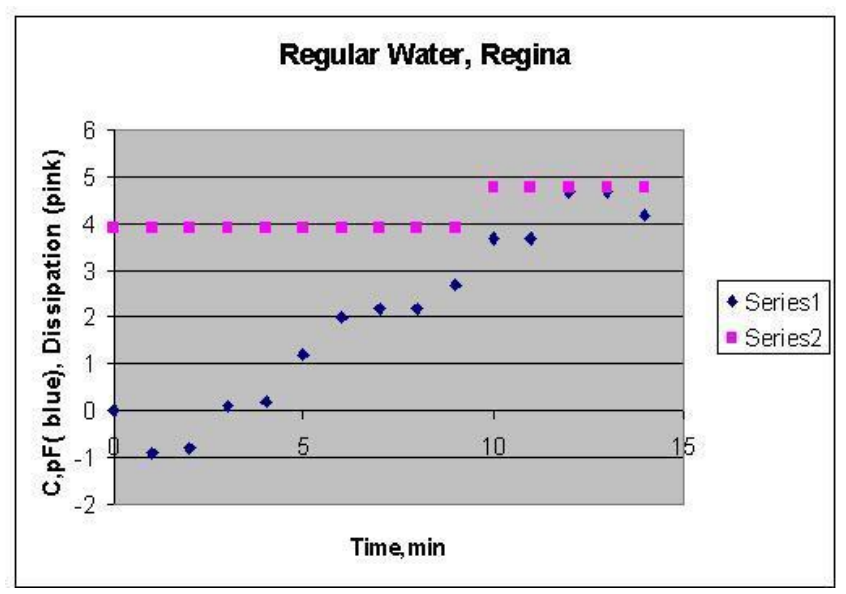

Fig.5. Initial capacitance $=93.2 \mathrm{pF}$ 
The Comparative Estimation of MRET Treated and Regular Water Absorption by Human Tissue with the Electro Physical Method

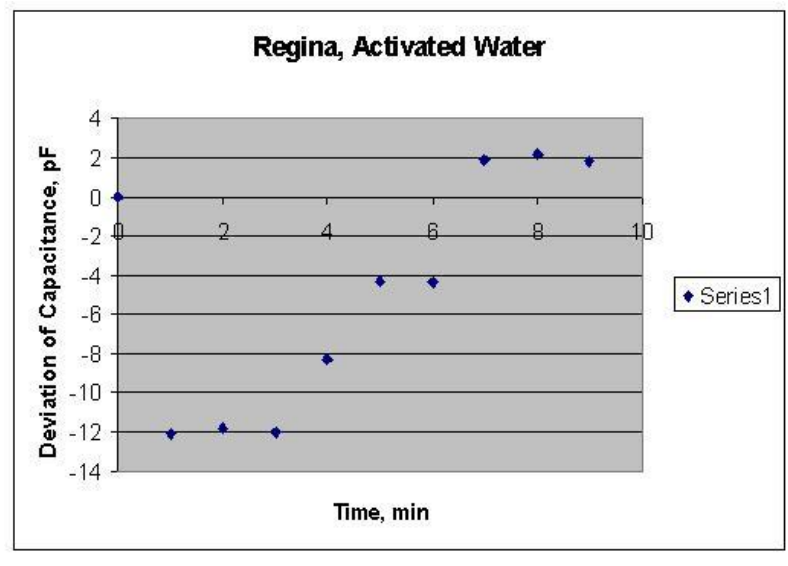

Fig.6. Initial capacitance $=95.9 \mathrm{pF}$

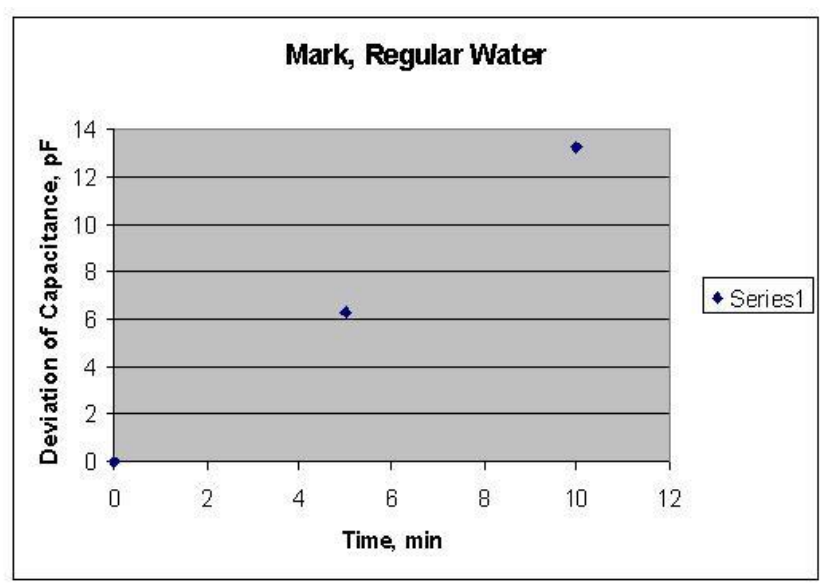

Fig.7. Initial capacitance $=101.0 \mathrm{pF}$.

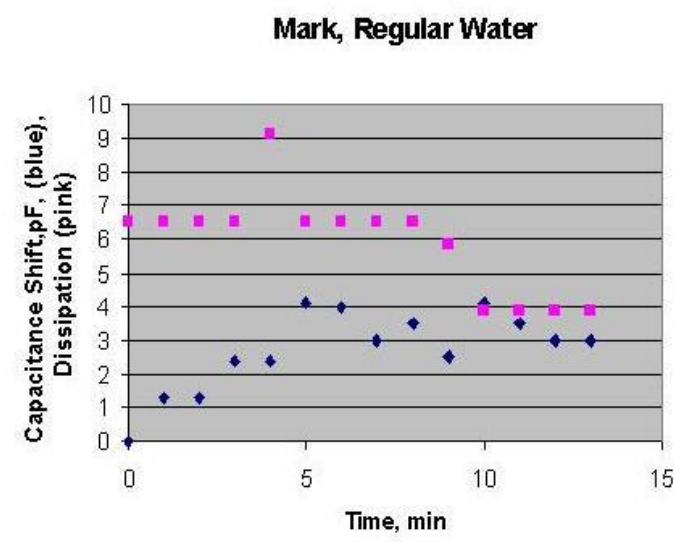

Fig.8. Initial capacitance $=102.5 \mathrm{pF}$

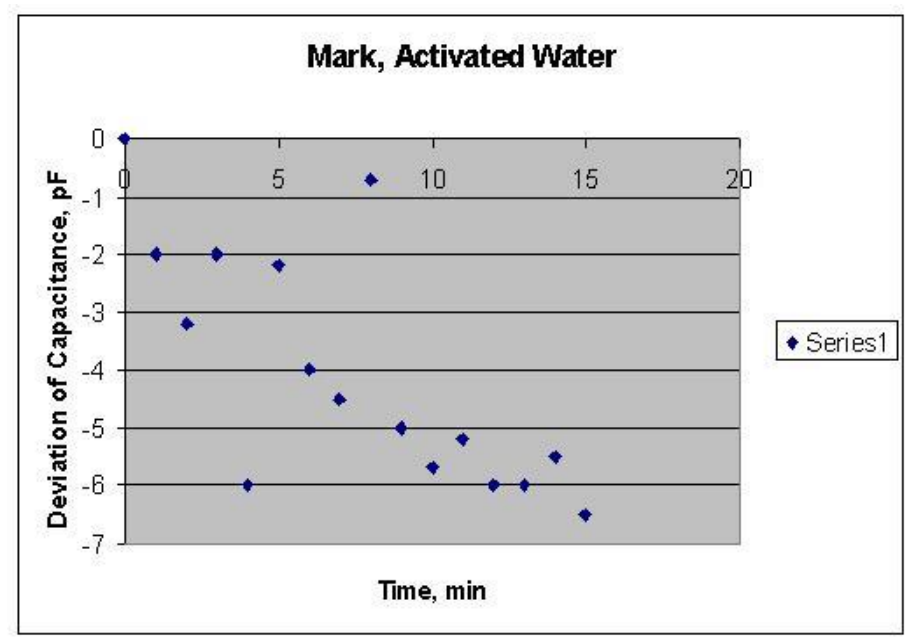

Fig.8. Initial capacitance $=113.2 \mathrm{pF}$

\section{Discussion:}

First of all, it has to be taken into consideration, that original water had $10 \mathrm{C}^{\mathrm{o}}$ temperature, while the temperature of the human body is $\sim 37 \mathrm{C}^{0}$. Therefore all the consumed water experienced a heating inside the human bodies. Therefore, we have to discuss a temperature dependence of the dielectric permittivity, $\frac{\partial \varepsilon}{\partial T}$ or its technical representative, $d C / d T$. The value is a considerable component of the entropy $S$. Both the free energy and the entropy are important indicators of surviving any system.

$A=A_{o}(T)+\frac{1}{2} \varepsilon_{0} \varepsilon E^{2}$

$S=S_{0}(T)+\frac{1}{2} \varepsilon_{0} \frac{\partial \varepsilon}{\partial T} E^{2}$

The role of $\frac{\partial \varepsilon}{\partial T}$ in the entropy of living organisms can not be overestimated. It's a coefficient before the value of $E^{2}$ ( $E$ - the field strength inside the objects, for instance -the living cells). Taking into consideration that $E$ inside the living cells can reach as order of

$10^{5} \mathrm{~V} / \mathrm{m}$ the $\frac{\partial \varepsilon}{\partial T}$ plays a special role. The systems with minimal entropy and free energy are more stable. Moreover, the entropy and free energy can predict a quality of functioning objects [1].

The results of the experiment have shown that drinking regular water causes increasing dielectric 
permittivity of the tissue, the positive $\frac{\partial \varepsilon}{\partial T}$, while drinking MRET activated water results in the negative value of $\frac{\partial \varepsilon}{\partial T}$. Therefore, the regular water increased the entropy of the tissue which makes it less stable, while the activated water reduces the entropy and makes the tissue more stable.

These results very well correlate with the findings of MRET activation effect on electrodynamic characteristics of water (complex dielectric permittivity and conductivity) conducted at the laboratory of Moscow State University, Russia. In this study, the significant modification of electrodynamic characteristics of distilled water was observed after MRET activation. The electrodynamic parameters of water as functions of applied external EMF frequencies are presented at Fig 9 (nonactivated water), Fig 10 (water activated for 30 minutes), Fig 11 (water activated for 60 minutes) and Fig 12 (30 minutes activated water heated up to $\left.72^{\circ} \mathrm{C}\right)$.

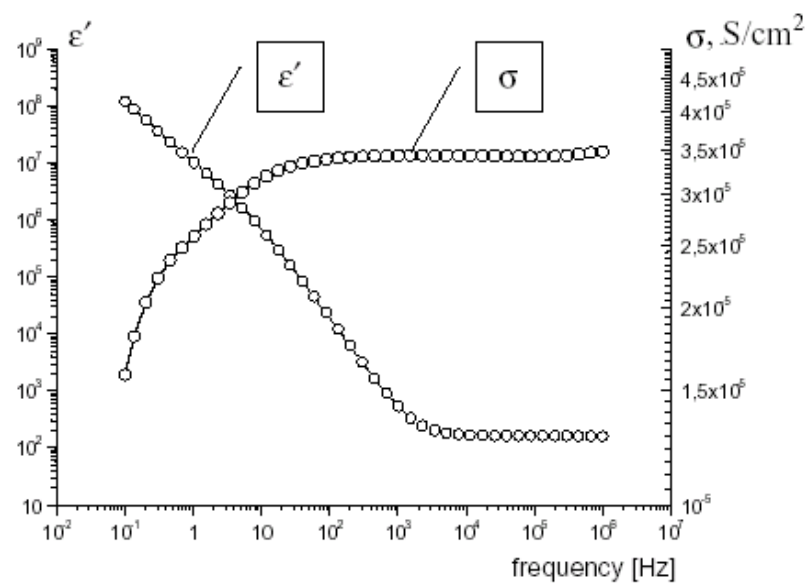

Fig 9: Electrodynamic characteristics of nonactivated distilled water at temperature $20^{\circ} \mathrm{C}$.

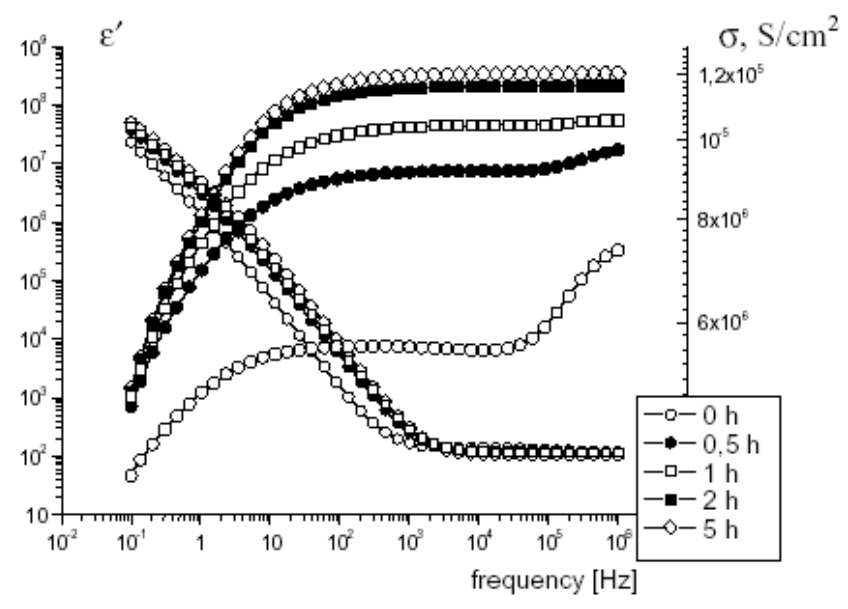

Fig 10: Electrodynamic characteristics of MRET water (30 minutes of activation) at temperature $20^{\circ} \mathrm{C}$ and different periods of time of storage: $0 \mathrm{~h}, 0.5 \mathrm{~h}$, $1.0 \mathrm{~h}, 2.0 \mathrm{~h}$, and $5.0 \mathrm{~h}$ respectively.

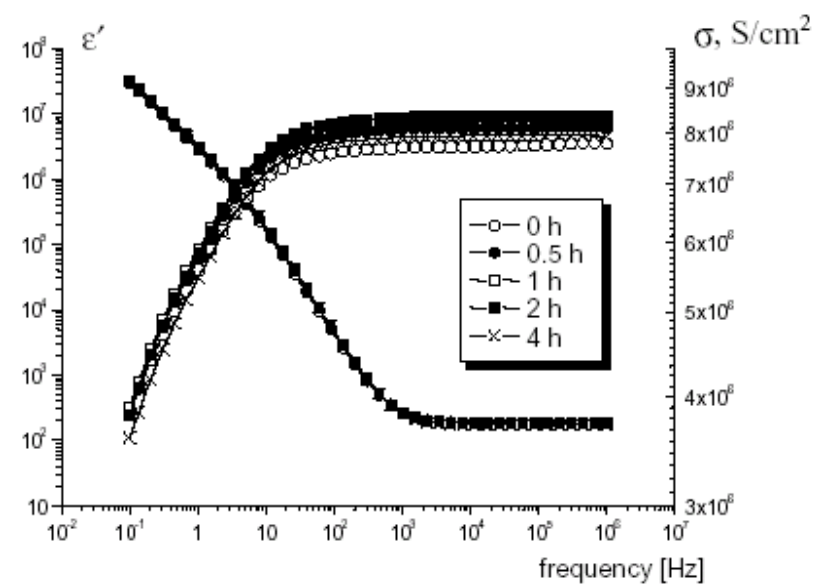

Fig 11: Electromagnetic characteristics of MRET water (60 minutes of activation) at temperature $20^{\circ} \mathrm{C}$ and different periods of time of storage: $0 \mathrm{~h}, 0.5 \mathrm{~h}$, $1.0 \mathrm{~h}, 2.0 \mathrm{~h}$, and $4.0 \mathrm{~h}$ respectively.

The dielectric permittivity in the very low frequencies range of $0.1-1000 \mathrm{~Hz}$ decreased by 80 $-\mathbf{9 0 \%}$ and in the range of frequencies of $1-100$ $\mathrm{kHz}$ it decreased by $18 \%$ in 30 minutes activated water; the decrease by $70-85 \%$ was observed in the range of $0.1-1000 \mathrm{~Hz}$ in 60 minutes activated water compared to non-activated water. The significant reduction of values of dielectric permittivity confirms the relatively high, long-range dynamic structuring of water molecules in activated water produced with the help of MRET activation process. The investigation regarding the electrodynamic characteristics of MRET water also revealed that the long-term storage of activated water (up to 5 hours at $20^{\circ} \mathrm{C}$ ) did not significantly affect the modified electrodynamic characteristics of $\mathbf{3 0}$ minutes activated water (the conductivity had the level of decrease $\mathbf{6 6 - 7 0 \%}$ and 
dielectric permittivity had the level of decrease $\mathbf{5 0}$ $\mathbf{5 5 \%}$ in the range of $0.1-1000 \mathrm{~Hz}$ and $18 \%$ in the range of $1-100 \mathrm{kHz}$ respectively). The storage of $\mathbf{6 0}$ minutes activated water under the same conditions practically did not affect its electrodynamic characteristics (maximum difference is $\mathbf{2 \%}$ ).

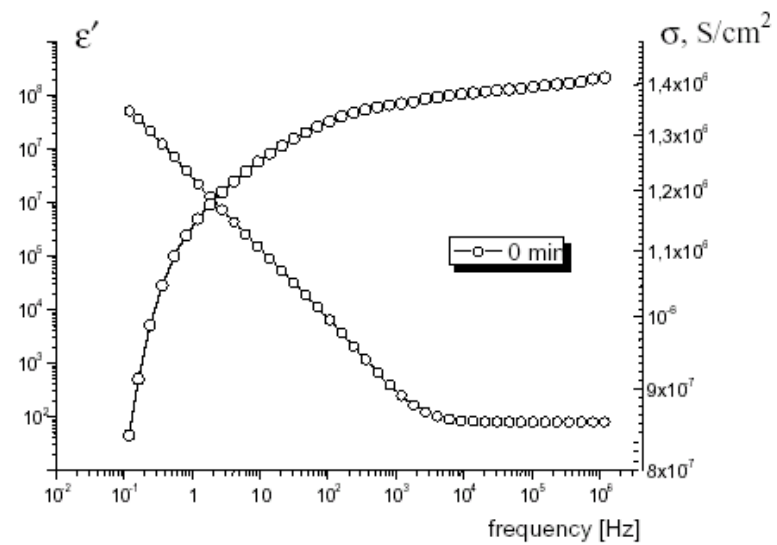

Fig 12: Electrodynamic characteristics of MRET water (30 minutes of activation) at temperature $72^{\circ}$ C.

The significant reduction of dielectric permittivity kept by MRET water activated for $\mathbf{3 0}$ minutes after it was heated to $\mathbf{7 2}^{\circ} \mathbf{C}$ confirms its stability to thermal effects. The reduction of dielectric permittivity of MRET water by 50-75\% in the range of $0.1-1000$ $\mathrm{Hz}$ (compare to non-activated water at $20^{\circ} \mathrm{C}$ ) provides the confirmation of the phenomenon of stability of MRET water to heating effect. According to [2] the reduction of dielectric permittivity due to the increase of water temperature is about $4.3 \%$ per $10^{\circ} \mathrm{C}$. It means that the reduction of dielectric permittivity due to the increase of water temperature was about $22 \%$ and the reduction of extra $38-43 \%$ most likely was related to the effect of MRET activation process. Such anomalous behavior of dielectric permittivity confirms the stability of MRET water to thermal effect [3].

Such anomalous stability of activated water electrodynamic characteristics to thermal effect and long time storage can be explained by entropy reduction of the physical system, in this case water molecular structure.

\section{A Seeming Paradox of Abnormally High Dielectric Permittivity of Water at Low Frequencies}

In the Figs.9-12 the real portion $\varepsilon$ ' of the dielectric permittivity of water looks abnormally high within $10^{-1}-10^{4} \mathrm{~Hz}$, yet looks frequency-descending.

The classical dielectric permittivity of water is 80.4 at $20^{\circ} \mathrm{C}$ from static fields up to hundreds of $\mathrm{MHz}$. The descending $\varepsilon^{\prime}(\mathrm{f})$ dependence is caused by relaxation processes in a near-electrode layer formed by a water-meal contact. The water-metal contact forms an Electrical Double Layer, EDL, having its own relaxation time $\tau$. Molecules of water are polar ones and polarization is an important component of forming EDL. The equivalent schematic of EDLbulk water system is shown in Fig.13.

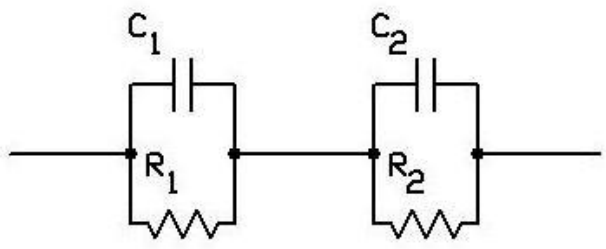

Fig.13. Equivalent circuit of a measuring cell taking into account the near-electrode EDL and the bulk water between the electrodes

Here, $C_{1} R_{1}$ represent EDL, while $C_{2} R_{2}$ represent a bulk water occupying most of the space between the electrodes, and finally can be reduced to one parallel $\mathrm{RC}$ circuit.

The admittance of the equivalent system is

$Y=g+i b=\frac{1}{R}+i \omega C$

According to general theory of dielectric dispersion in two-layer systems [5], the efficient capacitance of this system is

$C_{\text {eff }}=\frac{b}{\omega}=\frac{\tau_{1}+\tau_{2}-\tau+\tau_{1} \tau_{2} \tau \omega^{2}}{R_{0}\left[1+(\omega \tau)^{2}\right]}$

Here: $\tau_{1,2}$-relaxation times of layers 1 and $2, \tau$ is a system relaxation time, $R_{o}$ is a DC resistance of the effective capacitor.

According to [5], the system relaxation time $\tau$ is

$\tau=\frac{\tau_{1} R_{2}+\tau_{2} R_{1}}{R_{0}}$

As it follows from (7), value of $C_{\text {eff }}$ can be much higher at low frequencies than at the high frequencies. This results in a seemingly high, frequency-descending $\varepsilon^{\prime}$ at lower frequencies because traditional calculations of $\varepsilon^{\prime}$ are based on the total distance between the electrodes rather than that of EDL. The seeming phenomenon of high $\varepsilon^{\prime}$ at low frequencies is mostly related to behavior of the EDL rather than to the bulk water between the electrodes.

However, the MRET phenomenon affects both the near electrode EDL layers as well as the bulk portion of the water. 
The Comparative Estimation of MRET Treated and Regular Water Absorption by Human Tissue with the Electro Physical Method

\section{Conclusion}

- The complex dielectric permittivity method enables to study the dynamics of water absorption;

- The regular water makes $\frac{d \varepsilon}{d t}$ and $\frac{\partial \varepsilon}{\partial T}$ of the tissue positive and increases the entropy;

- The activated water makes $\frac{d \varepsilon}{d t}$ and $\frac{\partial \varepsilon}{\partial T}$ of the tissue negative and reduces the entropy.

\section{Reference:}

1. M. Krinker (2016) "The Experience of Predicting the Quality of Functioning Objects”, Journal of Unconventional Science. Vol. 12.

2. Chaplin, M. (2005) "Water Dielectric and Microwave Radiation”, London South Bank University, http://www.lsbu.ac.uk/water/microwave.html

3. Smirnov, I. (2008) "The Anomalous Electrodynamic Characteristics and Polarized- Oriented Multilayer Molecular Structure of MRET Activated Water", International Journal of Nanoscience, vol.7, 1-5.

4. https://en.wikipedia.org/wiki/Double_layer_(surface science)

5. P.T. Oreshkin (1977), Physics of Semiconductors and Dielectrics, Moscow, Russia. 